\title{
Assessment of Linear and Nonlinear/Complex Heartbeat Dynamics in Subclinical Depression (Dysphoria)
}

\author{
Alberto Greco ${ }^{1, *}$, Simone Messerotti Benvenuti ${ }^{2}$, Claudio \\ Gentili $^{2}$, Daniela Palomba ${ }^{2}$, Enzo Pasquale Scilingo ${ }^{1 * *}$, and \\ Gaetano Valenza ${ }^{1 * *}$ \\ ${ }^{1}$ Computational Physiology and Biomedical Instruments group, Bioengineering and \\ Robotics Research Center "E. Piaggio" and Dept. of Information Engineering, School \\ of Engineering, University of Pisa, Largo Lucio Lazzarino 1- 56122, Pisa, Italy. \\ ${ }^{2}$ Department of General Psychology, University of Padua - 35131 Padova, Italy \\ E-mail: * Corresponding author. Email: \\ alberto.greco@centropiaggio.unipi.it \\ ${ }^{* *}$ Senior Authors
}

\begin{abstract}
.
Depression is ranked as one of the leading causes of disability worldwide. Most of the previous studies focused on Major Depression, whereas studies on subclinical depression, such as the so-called dysphoria, have been overlooked. Indeed, dysphoria is associated with a high-prevalence of developing somatic disorders, and a reduction of quality of life and life-expectancy. In the current clinical practice, dysphoria is assessed using psychometric questionnaires and structured interviews exclusively, therefore without taking into account objective pathophysiological indices. To this extent, in this study we investigated heartbeat linear and nonlinear dynamics to derive objective autonomic nervous system biomarkers of dysphoria. Sixty undergraduate students participated in the study: according to the clinical evaluation, 24 of them were considered as dysphoric. Extensive group-wise statistics were performed to characterize the pathological vs. control groups. Moreover, a recursive feature elimination algorithm based on a $K$-NN classifier has been carried out for the automatic recognition of dysphoria at a single-subject level. Results showed that the most significant group-wise differences refers to increased heartbeat complexity (particularly for fractal dimension, sample entropy and recurrence plot analysis) w.r.t. healthy controls, confirming dysfunctional nonlinear sympatho-vagal dynamics in mood disorders. Furthermore, a balanced accuracy of $79.17 \%$ has been achieved in automatically discerning dysphoria patients from controls, with the most informative power to nonlinear, spectral and polyspectral quantifiers of cardiovascular variability. This study experimentally supports the assessment of dysphoria as a defined clinical condition with specific characteristics which are different both from healthy fully euthimic controls and from full-blown major depression.
\end{abstract}




\section{INTRODUCTION}

Depression is a commonly occurring, invalidating and recurrent disorder which causes deterioration of quality of life, somatic morbidity, and mortality [1]. Although epidemiological data is not available for many countries, current statistics indicate that depression is widespread throughout the world with a high-variability in prevalence [1,3]. Indeed, the World Health Organization has ranked depression as the 4th leading cause of disability worldwide [4] and projects that, by 2020, it will be the 2nd [5]. Importantly, people with depressive disorder have an increased risk of developing severe somatic diseases, including diabetes and cardiovascular diseases [6, 8-13].

In the current clinical practice, Major Depression is typically diagnosed following the Diagnostic and Statistical Manual of Mental Disorders (DSM-5) criteria, edited by the American Psychological Association [14]. Specifically, clinicians ascertain the presence of depressed mood or anhedonia, along with a minimum additional four out of nine diagnostic criteria (symptoms), such as irritability, decreased interest or pleasure in most activities, significant weight change, change in sleep patterns, and fatigue.

Major criticisms of this approach are as follows:

i) clinical decision making: the use of very precise cut-off values to discern clinically relevant conditions from not relevant ones according to subjective, patient-reported answer to structured questions. For example, depressive symptoms are commonly assessed using clinician-administered rating scales and questionnaires, such as Hamilton Rating Scale for Depression [29] or self-administered rating scales, such as the Beck Depression Inventory-II (BDI-II) [31]. In the Italian version, a BDI-II score of 12 has been reported as the optimal threshold to discern individuals with and without clinically significant depressive symptoms [31] and to be predictive of a diagnosis of Major Depression with the Structured Clinical Interview from DSM [32].

ii) minimum number of symptoms: in case of Depression, patients cannot be diagnosed if they report less than five symptoms, despite the clinical severity of each symptom perse.

iii) clinical heterogeneity: depression is a heterogeneous disorder with more than 100 different combination of symptoms fulfilling the DSM criteria [15]. Routine clinical evaluation, based on subjective retrospective recollection of mood symptoms during the previous two weeks, may be biased by a well-known memory distortions present in depressed patients. They, in fact, tend to rehearse negative memories better than positive ones, leading to an overestimation of the length and severity of their symptoms [18].

iv) low degree of diagnostic consensus: there is a very low-degree of diagnostic consensus in DSM-5 diagnosis. The $k$ agreement on depression diagnosis with DSM-5 criteria, in fact, is very low (0.2 in the field tests performed in US and Canada) [16].

As reported in several epidemiological studies, the above-mentioned limitations are emphasized in case of depressive mood alterations that do not completely fulfil the DSM-5 criteria for Major Depression. Exemplarily, the so-called dysphoria or Minor 
Depression shows similar risks for somatic disorders and similar reduction of quality of life and life expectation than major depression [19-22]. Clinically, dysphoria has been defined as the presence of at least two, but not more than four, symptoms of Depression listed in the DSM-5 [23-28]. In this view, dysphoric patients have a clinical severity that may be comparable to patients with Major Depression but with a narrowed symptomatology spectrum.

It is evident that diagnosis of mental disorders seriously lacks of objective psychophysiological measurements, and/or biochemical markers to be taken into account. Major research attempts indeed tried to overcome this limitation. Focusing on the assessment of Autonomic Nervous System (ANS) activity on cardiovascular control, previous research efforts have shown a strong link between ANS dysfunctions and depressive symptoms [33-37]. Of note, many ANS activity indices are quantified through linear and nonlinear analysis of heart rate variability (HRV) series, resulting from the dynamical balancing of the sympathetic and parasympathetic branches.

Studies on HRV series have associated depressive symptoms with reduced parasympathetic activity with respect to healthy conditions [17,38-40], as estimated through frequency-domain estimates within the high-frequency (HF) band (0.15-0.4 $\mathrm{Hz}$ ). Significant results have been achieved considering that combined sympathetic and vagal stimulation on heart rate is not simply additive [2], due to interplay with numerous other physiological subsystems (e.g., endocrine, neural, and respiratory), as well as multiple self-regulating, adaptive biochemical processes. To this extent, HRV measures derived from the theory of nonlinear system dynamics have been successfully employed to increase the specificity of HRV-based decision support for depression [38,41-45]. Specifically, heartbeat complexity changes have been investigated using entropy measures, high order spectra, recurrence plots and Poincaré geometry [46-48,52]. Nevertheless, none of these approaches has been profitably used for the assessment of dysphoria, therefore objective ANS markers of such minor depressive symptoms are still unknown.

To overcome this limitation, in this study we aim to exploit measures of heartbeat linear and nonlinear dynamics for the assessment of dysphoria in young adults. Specifically, besides classical group-wise statistical analysis to identify the most effective HRV measures discerning dysphoric and non-dysphoric subjects, to increase the specificity our approach and push onto a direct clinical application, we propose the use of k-Nearest Neighbour $(K-\mathrm{NN})$ algorithms to automatically identify dysphoric subjects at a single-subject level. Importantly, we have limited our research to adult females to avoid possible confounding factors due to gender. In fact, the higher prevalence of major depression in females than males has been consistently reported in the general adult population [53-58], confirming the strong gender effect on the spread of depressive symptoms.

Methodological details, avoiding the description of all the well-known and widelyreported HRV metrics, as well as experimental results, and Conclusion and Discussions follow below. 


\section{METHODS}

\subsection{Subject Recruitment and Acquisition set-up}

The present study was carried out with the adequate understanding and written consent of the participants in accordance with the Declaration of Helsinki. The study was approved by the Ethics Committee of the Department of General Psychology, University of Padua (Italy).

Sixty volunteers (average age of $21.89 \pm 2.06$ ) were enrolled in this study. All subjects were female undergraduate students from the University of Padua. To ensure the validity of this study, none of the participants were taking antidepressants or medications known to affect the ANS functioning or have had a history of neurological or cardiovascular diseases or alcohol use disorders. In addition, participants were asked to avoid drinking coffee for at least $2 \mathrm{~h}$ before the experiment starts.

The experimental protocol comprised a 5-min continuous electrocardiogram (ECG) recording in resting state, according to the current clinical evaluation in cardiology. Each volunteer was asked to comfortably seat on an armchair in a sound-attenuated, dimly-lit room. The ECG was recorded using $\mathrm{Ag} / \mathrm{AgCl}$ surface electrodes positioned on the participant's chest in a modified lead II configuration. The ECG signal was amplified with a gain of 150 , digitized at $500 \mathrm{~Hz}$ (16 bit A/D converter; resolution $0.559 \mu \mathrm{V} / \mathrm{LSB}$ ), and stored on to a Pentium IV computer. In order to avoid movement artifacts, participants were instructed to stay still and not to talk throughout the ECG recordings.

Before the ECG acquisition, psychometric tests were administered. Each subject filled out the following tests:

- the BDI-II questionnaire [30,31] to assess possible depressive symptoms. This test is a reliable and valid self-report questionnaire that evaluates the severity of symptoms of depression over the past 2 weeks. Answers are given on a four-point (0-3) Likert scale and scores range from 0 to 63 , with higher scores indicating more severe depressive symptoms. As mentioned before, in the Italian version, a score over 12 detects individuals with problems of depression in the Italian population [31].

- The State and Trait Anxiety Inventory (STAI) test, consisted of two questionnaires (STAI-Y1 for state anxiety and STAI-Y2 for trait anxiety), both consisting of 20 multiple-choice items [61]. This test is based on the conceptual distinction between "state" and "trait" anxiety this test makes possible to distinguish between anxiety as a transitory state and anxiety as a relatively stable personality trait.

- The Emotion Regulation Questionnaire (ERQ) [62] consisted of a 10-item scale designed to measure subjects' tendency to regulate their emotions in two ways: cognitive Reappraisal and expressive Suppression. Participants answer each item on a 7 -point Likert-type scale ranging from 1 (strongly disagree) to 7 (strongly agree).

In addition, subjects who exceeded the BDI-II cut-off of 12 were administered the 
mood episode module (module A) of the SCID-I [32] by a trained psychologist, in order to confirm the presence of dysphoria and to exclude those participants who met the diagnostic criteria for major depression, dysthymia or mood disorders.

After filling out the psychometric tests, the participants answered to an ad-hoc interview that provided information on their age, health status, smoking habits, and regular alcohol use.

The enrolled subjects were identified within a group of 224 undergraduate students from the University of Padua that completed an online version of the BDI-II. Only subjects who achieved a BDI-II score equal to or less than 8 or equal to or greater than 12 were enrolled in the experiment. More specifically, individuals who scored at least 12 on the BDI-II test and had two to four depressive symptoms for at least two weeks, were assigned to the dysphoric group. Conversely, undergraduates who scored equal to or less than 8 (corresponding to the 53th percentile) on the BDI-II, and showed no depressive symptoms as defined by the SCID-I were identified as healthy controls. According to the BDI-II scores, the volunteers were grouped as dysphoric and non-dysphoric subjects. The dysphoric group comprised 24 participants, whereas the non-dysphoric group comprised 36 students. Characteristics of both groups are shown in Table 1.

\subsection{Heart Rate Variability Linear and Nonlinear Measures}

To derive HRV series, ECG signals were band-pass filtered (0.05-40 Hz) to reduce noise and motion artifacts.

The R-peaks were detected using the Pan-Tompkins algorithm [63]. In order to correct possible physiological (e.g., ectopic beats) or algorithmic (e.g., R-peak misdetection) artefact in the $\mathrm{RR}$ time series, a recently proposed real-time $\mathrm{R}-\mathrm{R}$ interval error detection and correction algorithm based on point-process statistics (prediction log-likelihood) was applied [64]. In addition all the segments were visually inspected.

Standard HRV metrics aims at quantifying cardiovascular linear dynamics through time and frequency domain estimates [65]. More specifically, within a given time

Table 1. Participant characteristics represented by their Median value (25th, 75th, percentile) of each group.

\begin{tabular}{|c|c|c|c|}
\hline Variables & Whole group & Controls $(36)$ & Dysphoric $(24)$ \\
\hline Age (years) & $22(20,24)$ & $22(20,24)$ & $21(20,22.5)$ \\
Education (years) & $16(14,17)$ & $16(15,17)$ & $15(14,17)$ \\
STAI-Y1 & $33(30,36)$ & $31.5(29.5,34.5)$ & $34(32,40)$ \\
STAI-Y2 & $39.5(33.5,52)$ & $35(31,39)$ & $52.5(49,61.5)$ \\
BDI-II & $7(2,13.5)$ & $2.5(1,5.5)$ & $14,(12.5,20.5)$ \\
ERQ Reappraisal & $31(25.5,33.5)$ & $31(29,35)$ & $29.5(23,32.5)$ \\
ERQ Suppression & $13(8.5,17)$ & $12(7.5,15.5)$ & $14.5(11,17)$ \\
\hline
\end{tabular}


window, we calculated the mean value $\left(\mu_{R R}\right)$ and the standard deviation $\left(\sigma_{R R}\right)$ of the RR intervals, along with the square root of the mean squared differences between successive RR intervals (RMSSD). In the frequency domain, the HRV spectra were calculated using Welch's periodogram method with 50\% data overlap. A Blackman window (256s) was applied to each segment and the fast Fourier transform was calculated for each windowed segment. Finally, the power spectra of the segments were averaged. To this aim, the non-evenly sampled RR interval series were interpolated by means of a cubic spline functions. We then calculated the power within the three main spectral bands: Very Low Frequency band (VLF, below $0.04 \mathrm{~Hz}$ ), Low Frequency band (LF, from $0.04 \mathrm{~Hz}$ to $0.15 \mathrm{~Hz}$ ), and High Frequency band (HF, from $0.15 \mathrm{~Hz}$ to $0.4 \mathrm{~Hz}$ ). In addition, the ratio between the LF and the HF power was computed.

In addition, HRV nonlinear/complexity measures were derived using wellknown procedures such as detrended fluctuation analysis (DFA $\alpha_{1}$ ), sample entropy (SampEn), fractal dimension (FracDim), Lagged Poincaré Plot (LPP), and Recurrence Quantification Analysis (RQA) [65]. DFA investigates the statistical self-affinity and correlations over HRV series, more specifically we evaluated the short-term fluctuations characterized by the slope $\alpha_{1}$ (obtained using RR segments of length 16). The SampEn quantifies the time series regularity and predictability (we adopetd a tolerance parameter of 0.2). FracDim evaluates the fractal characteristics of cardiovascular variability (we applied the method reported in [69]). RQA refers to the study of the Recurrence Plot, providing measures as the Recurrence Rate (RecurrRate), i.e., the percentage of recurrence points in an RP, and the Determinism (DET), which is the percentage of recurrence points which form diagonal lines. Of note, to build the RP matrix, we used the canonical threshold distance of $\sqrt{m} \cdot \sigma_{R R}$, where $m=10$ is the embedding dimension $[48,49]$. LPP quantifies HRV fluctuations through two standard deviation, $\mathrm{SD} 1$ and SD2, and their ratio (SD12), of a M-lag scatter-plot of RR intervals. In addition, we computed the SDRR index, i.e, an approximate relation indicating the variance of the whole HRV series (see [50] for details).

Finally, Higher-Order Spectra (HOS) [65] parameters were also computed. HOS of HRV series refer to Bispectal analysis, i.e., the two dimensional Fourier Transform of the third order cumulant [65], deriving the Phase Entropy (PhaseEntr) and the mean magnitude (MeanMagnitude) of the bispectrum, as well as nonlinear sympatho-vagal interactions obtained by integrating the Bispectral plane in the appropriate frequency bands: LL $\left(f_{1}:(0-0.15] \mathrm{Hz}, f_{2}:(0-0.15] \mathrm{Hz}\right), \mathrm{LH}\left(f_{1}:(0-0.15] \mathrm{Hz}, f_{2}:(0.15-0.4] \mathrm{Hz}\right), \mathrm{HH}$ $\left(f_{1}:(0.15-0.4] \mathrm{Hz}, f_{2}:(0.15-0.4] \mathrm{Hz}\right)$.

\subsection{Statistical and Correlation Analyses}

For each HRV feature, the Shapiro-Wilk test was applied to verify the null-hypothesis normally-distributed population. In case of non-Gaussian distribution, a non-parametric Mann-Whitney U test was adopted to statistically compare the two groups. In order to address the problem of multiple comparisons, we control the false discovery rate (FDR). 
More specifically, we adopted the Bejnamini \& Hochberg [51] algorithm which controls the FDR at a level of 0.05. Additionally, a non-parametric correlation analysis, based on Spearman coefficient, between each HRV feature and correspondent BDI-II scores was performed.

\subsection{Pattern Classification}

A pattern recognition analysis, including feature selection, was carried out to increase the specificity of the proposed HRV-based approach. In the machine learning context, feature selection methods can be divided in two main categories: (i) filter methods, which focus in finding dependences between features (using e.g., correlation index, information measure, statistical test, Fisher's criterion) that affect the accuracy level without involving the selected learning algorithm; (ii) wrapper methods, which instead assess subset selection of features according to their usefulness to a given specific predictor and use this latter as the evaluation function. Here, we tested and compared two filter approaches based on statistics and correlation analysis and two wrapper methods applied to $K-N N$ and SVM learning algorithm. Classification performance for each subset of selected features is computed following a recursive feature elimination ( $R F E$ ) procedure.

2.4.1. K-NN-RFE: We applied a Recursive Features Elimination (RFE) algorithm to a $K$-NN classifier, which was validated through a leave one subject out (LOSO) crossvalidation procedure. $K-\mathrm{NN}$ is a supervised non-parametric classifier. Given a query observation $x_{0}$, and a labeled training-set, it finds the $K$ training points $x(r), r=1, \ldots, k$ closest in (Euclidean) distance to $x_{0}$, and then classify it according to the most frequent class among the $K$-neighbors.

Following the LOSO scheme, $K$-NN parameter (i.e, number of neighbors) and feature ranking were automatically tuned (Figure 1).

Particularly, according to the LOSO procedure, we iteratively split the dataset into a training-set and a test-set. Each training-set was comprised of the observations from $(N-1)$ subjects (where $N$ is the total number of participants), and each test-set was comprised of the observation from the $N_{t h}$ left-out subject. This procedure was iterated $N$ times. For each of the $N$ iterations the test-set observations were classified by the RFE- $K$-NN classifier using the parameter $K$ and the feature ranking estimated within the training-set. More specifically, HRV features were ranked according to the following procedure comprised of $M$ steps (note that $M$ is the total number of the features):

1) The first step selected the highest-ranking position. Within the training set, $M$ classifications were performed using only a single feature. The feature which achieved the highest accuracy (i.e., highest predictive power, PP) was associated with the highest-ranking position. Of note, each of the $M$ classifications was 
performed following a nested-LOSO procedure based on a $K$-NN classifier with the same approach explained above. More in detail, the training-set was in turn divided in a nested-training-set, comprised of the observations from $(N-2)$ subjects, and a nested-test-set comprised of the observations of the left-out subject among those $(N-1)$ of the training set.

2) The second step selected the 2nd-ranking position. Each one of the remaining $(M-1)$ features was iteratively added to the first selected feature, generating $(M-1)$ bi-dimensional feature-sets. For each of the $(M-1)$ iterations, we calculated the classification accuracy of each new bi-dimensional feature-set following, also in this case, a nested-LOSO procedure. The feature which, together to the one selected at the previous step, achieved the highest accuracy (i.e., highest cumulative predictive power, CPP) was associated with the 2nd-ranking position;

Q) The Qth step selected the Qth-ranking position. Likewise step 2, each one of the remaining $(M-(Q-1))$ features was iteratively joined to the $(Q-1)$ previously selected feature, generating $(M-(Q-1)) Q$-dimensional feature-sets. The feature which, together to the $(Q-1)$ previously selected ones, achieved the highest accuracy (i.e., highest CPP) was associated with the Qth-ranking position; $\vdots$

M) The last remaining feature occupied the last ranking-position.

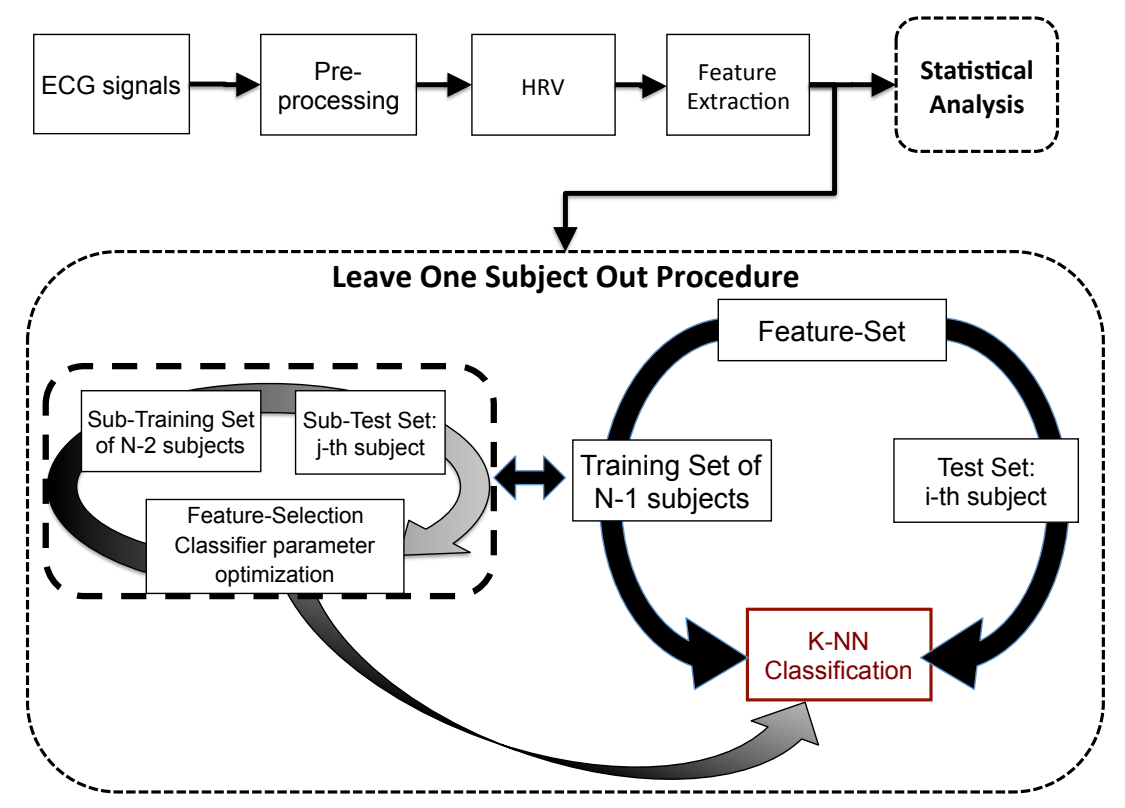

Figure 1. Scheme of the K-NN-RFE classification procedure. ECG signal is processed in order to estimate the HRV time series and extract several linear and nonlinear features. The feature-set is used as input of a twostage leave-one-subject-out procedure based on a K-NN classifier and a feature selection algorithm. 
This $M$-step feature-ranking procedure was in turn repeated increasing at each repetition the number of neighbors $K$. The optimal $K$ was the one that achieved the highest CPP.

This robust method allows an unbiased out-sample error estimate, reducing the risk of overfitting.

Once that the $K$ parameter and the associated feature ranking were estimated, the $K$-NN RFE classified the test-set performing a dimensionality reduction by recursively pruning the least important feature from current feature-set, according to the ranking. Result section will show the average accuracy of all RFE stages and results of the most accurate classification, using an optimal feature set, are shown in terms of confusion matrix. Values on the diagonal of this $2 \mathrm{X} 2$ table represent the percentage of subjects that were correctly associated as belonging to a specific class (true positives and true negatives).

2.4.2. SVM-RFE, K-NN-STAT-RFE，SVM-STAT-RFE，K-NN-CORR-RFE, and $S V M-C O R R-R F E$ : To compare with a more standard approach, performances of the proposed $K$-NN-RFE algorithm were compared with a standard SVM-RFE algorithm [71], as well as with two filter-approach alternatives, i.e., STAT-RFE and CORR-RFE.

Specifically, SVM-RFE employed a nu-SVM $(\mathrm{nu}=0.5)$ with a radial basis kernel function with $\gamma=M^{-1}$, where $M=26$ is the number of HRV features (for further details concerning the RFE criteria see [71]). The STAT-RFE and CORR-RFE. adopted a recursive feature elimination algorithm applied to both $K-N N$ and $S V M$ classifiers based on p-value and correlation coefficient. respectively.

The recursive algorithm followed the same approach explained in section 2.4.1. However, at each iteration of the LOSO scheme, the features were ranked relying on the statistical comparison between the dysphoric and nondysphoric groups (K-NN-STAT-RFE and SVM-STAT-RFE) and on correlation between each feature and the classes (K-NN-CORR-RFE, and $S V M-C O R R-R F E)$. More specifically, the features were ranked according to the associated p-value (in ascending order).

\section{RESULTS}

Results from the group-wise statistical analysis between the dysphoric and nondysphoric groups are shown in Table 2. Asterisks show the statistical significant features. Importantly, dysphoric patients showed a significant increase of HRV complexity as estimated through fractal dimension and sample entropy. Likewise, features extracted from LPP, excluding SD1, significantly increased in the dysphoric group. A similar trend was also shown from $\mu_{R R}, \sigma_{R R}$, and LF and HF power, estimating HRV linear dynamics. Two parameters from RQA, i.e., Recurrence Rate and Determinism, resulted significantly decreased in dysforic subjects with respect to healthy ones. 


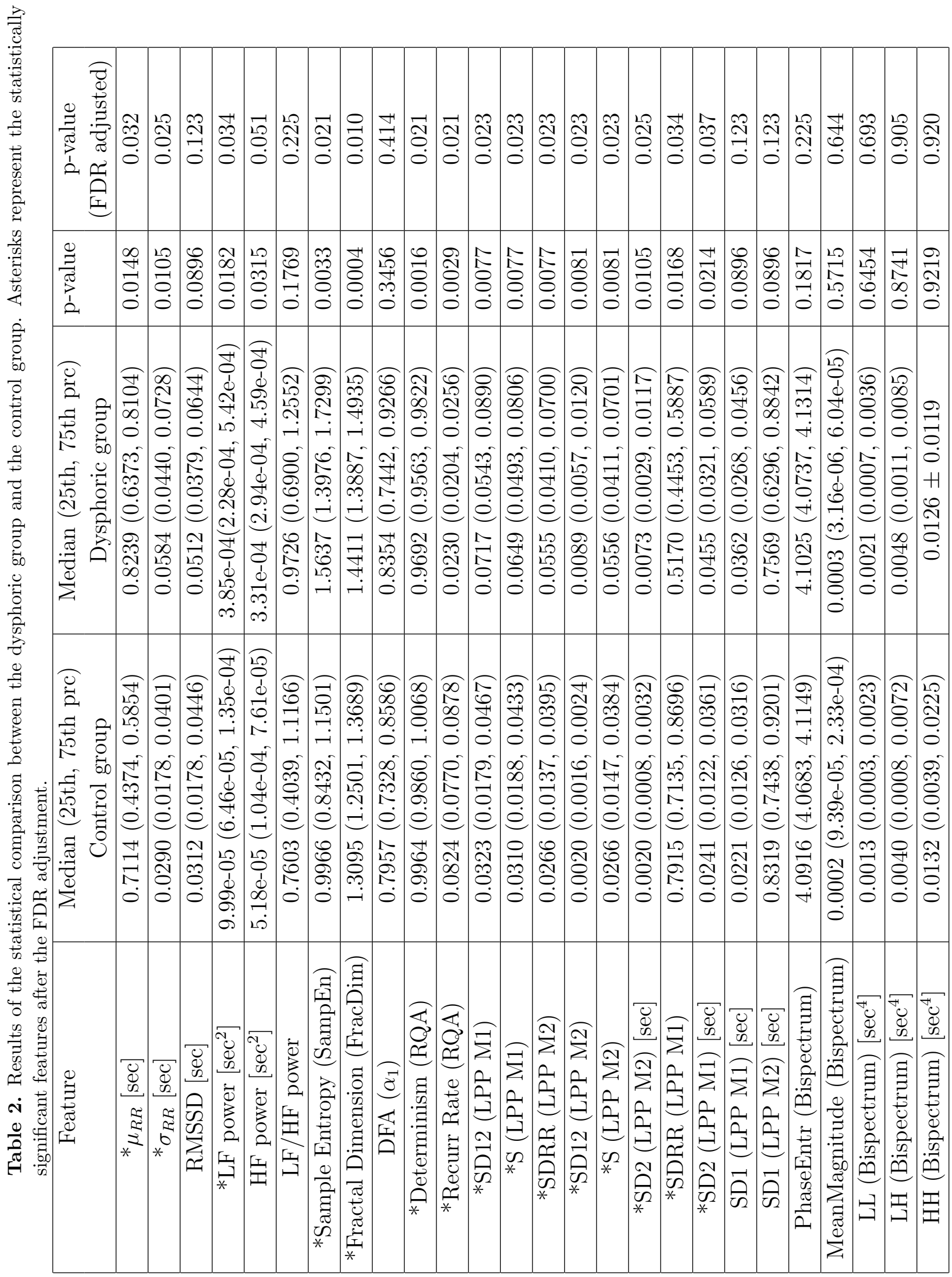




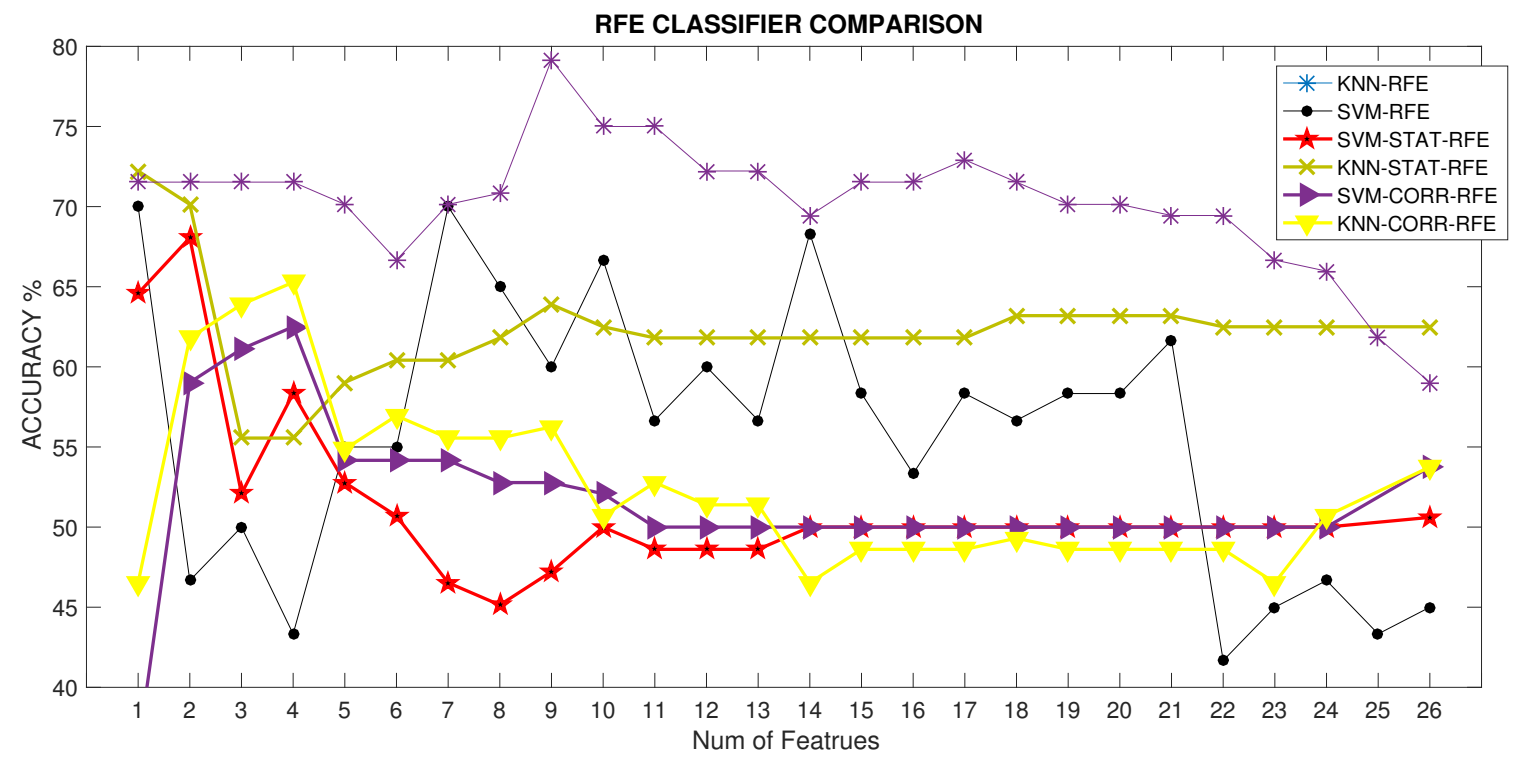

Figure 2. Accuracy trend on validation-set as a function of the number of selected features. For each method, the corresponding curve shows the accuracy considering the first $\mathrm{N}$ ranked features (from 1 to 26). Features are ranked according to the corresponding algorithm criterion.

Concerning the correlation analysis, no significant differences were found, except for the SDRR feature that correlated with the BDI-II scores of the control group with a $\rho$ of 0.51 .

Concerning the classification, Figure 2 shows the balanced accuracy (i.e., average between specificity and sensitivity of the confusion matrix) at each iteration of the proposed $K$-NN-RFE algorithm, as well as the other classifiers. Note that $K$-NN-RFE outperformed SVM-STAT/CORR-RFE, $K$-NN-STAT/CORR-RFE and the canonical SVM-RFE, and achieved a maximum balanced accuracy of $79.17 \%$, with sensitivity $75.00 \%$ and specificity $83.33 \%$, respectively. The maximum accuracy was achieved selecting the nine most relevant features according to the RFE criteria.

The complete list of features ordered by their median rank over all folds is reported in Table 3. Note that the most informative feature was the Fractal Dimension, and 6 out of the 9 selected features were derived from heartbeat nonlinear/complex dynamics and HOS analysis.

To further demonstrate the crucial role of such nonlinear dynamics as additional biomarker of dysphoria, we performed a $K$-NN classification considering linear and nonlinear HRV features separately. Note that, in both cases, the proposed $K$-NN-RFE algorithm, showed a peak in the balanced accuracy using the first 3 most informative features. However, in the linear case we achieved a balanced accuracy of $66.67 \%$ (Table 5), while in the nonlinear case, the balanced accuracy reached the $75.70 \%$ (Table 6 ). 
Table 3. Features ranking according to the highest cumulative predictive power (see Section 2.4.1).

\begin{tabular}{|cc|}
\hline Feature & \\
& $*$ \\
\hline FractDim & $*$ \\
\hline LF power & $*$ \\
\hline HF power & $*$ \\
\hline MeanMagnitude (Bispectrum) & $*$ \\
\hline LL (Bispectrum) & $*$ \\
\hline PhaseEntr (Bispectrum) & $*$ \\
\hline Recurr Rate (RQA) & $*$ \\
\hline RMSSD & $*$ \\
\hline Determinism (RQA) & \\
\hline LH (Bispectrum) & \\
\hline SD2 (LPP M2) & \\
\hline SD1 (LPP M1) & \\
\hline SD12 (LPP M2) & \\
\hline S (LPP M1) & \\
\hline$\mu_{R R}$ \\
\hline$\sigma_{R R}$ \\
\hline SD1 (LPP M2) \\
\hline HH (Bispectrum) \\
\hline SD2 (LPP M1) \\
\hline S (LPP M2) \\
\hline SD12 (LPP M1) \\
\hline SDRR (LPP M2) \\
\hline SDRR (LPP M1) \\
\hline Sample Entropy \\
\hline LF /HF power \\
\hline DFA $\alpha_{1}$ \\
\hline
\end{tabular}

asterisks represent the selected features for the most accurate $K$-NN model.

Table 4. Confusion matrix obtained applying the $K$-NN-RFE method to the whole feature-set. The table corresponds to the feature selection step that achieves the highest accuracy (i.e., the maximum of the blue curve in Figure 2).

\begin{tabular}{|c|c|c|}
\hline$K-\boldsymbol{N} N$ & DYSPHORIC & NON-DYSPHORIC \\
\hline DYSPHORIC & $\mathbf{7 5 . 0 0 \%}$ & $16.67 \%$ \\
\hline NONDYSPHORIC & $25.00 \%$ & $\mathbf{8 3 . 3 3 \%}$ \\
\hline
\end{tabular}

Balanced accuracy: $79.16 \%$ (considering the first 9 features with the highest prediction power (see Table 3)). 
Table 5. Confusion matrix obtained applying the $K$-NN-RFE method to the linear feature-set only. The table corresponds to the feature selection step that achieves the highest accuracy.

\begin{tabular}{|c|c|c|}
\hline$K-\boldsymbol{N} N$ & DYSPHORIC & NON-DYSPHORIC \\
\hline DYSPHORIC & $\mathbf{5 8 . 3 3 \%}$ & $25.00 \%$ \\
\hline NONDYSPHORIC & $41.77 \%$ & $\mathbf{7 5 . 0 0 \%}$ \\
\hline
\end{tabular}

Balanced accuracy: $66.67 \%$ (considering the first 3 features with the highest prediction power: HF, LF, and RMSSD).

Table 6. Confusion matrix obtained applying the $K$-NN-RFE method to the Nonlinear/HOS feature-set only. The table corresponds to the feature selection step that achieves the highest accuracy.

\begin{tabular}{|c|c|c|}
\hline$K-\boldsymbol{N} N$ & DYSPHORIC & NON-DYSPHORIC \\
\hline DYSPHORIC & $\mathbf{7 0 . 8 3 \%}$ & $19.44 \%$ \\
\hline NONDYSPHORIC & $29.17 \%$ & $\mathbf{8 0 . 5 6 \%}$ \\
\hline
\end{tabular}

Balanced accuracy: $75.70 \%$ (considering the first 3 features with the highest prediction power: FracDim, MeanMagnitude (Bispectrum), and Determinism (RQA)).

\section{DISCUSSION AND CONCLUSION}

We investigated heartbeat linear and nonlinear/complex dynamics in dysphoric young female adults considering short-term ECG recordings in resting state. Sixty undergraduate students were enrolled in the study, including 24 dysphoric 36 control subjects with properly associated BDI-II scores. Our results demonstrate that it is possible to derive effective biomarkers of dysphoria using a proper combination of linear and nonlinear/complexity quantifiers of cardiovascular variability, as from HRV series. Furthermore, using this approach we were able to describe new psychophysiological characteristics of dysphoria which have not been described. Namely, as a preliminary/exploratory step, non-parametric statistical analysis revealed that most significant differences were associated with HRV complexity (especially fractal dimension and sample entropy), recurrence plot features, and LPP parameters, with higher values in dysphoria. As a large amount of literature links pathological (mental) states, including major depression [7], with reduced cardiovascular complexity $[7,42,65,72,73]$, our results pose several scientific questions for the understanding the neurobiology underpinning minor depressive disorders. Note that other cardiovascular and neurological pathologies, such as post-infarction [75] and Parkinson's disease [74] have been associated with increased cardiovascular complexity than healthy controls. To our knowledge, this is the first study assessing dysphoria in terms of multi-dimensional autonomic patterns, proposing a different and more complex biological scenario than depression. According to a relevant line of research, our data suggests that dysphoria represents a specific and distinct clinical entity [76, 77], and not simply a less severe form of depression [78]. 
Moreover, also epidemiological data regarding the morbility and mortality of this condition suggest that it has to be treated as a specific and clinically relevant entity [76, 77]. More studies (see below in the limitations), are needed to further confirm such an hypothesis.

Aiming to a direct clinical translation of these results, we moved beyond standard statistical analysis and developed an automatic decision support system algorithm to discern dysphoria from healthy controls using a recursive feature elimination procedure applied to a $K$-NN-based classifier $(K-\mathrm{NN}-\mathrm{RFE})$. This confirmed the significant discriminant power of heartbeat complexity, particularly referring to fractal dimension and RQA. In fact, by properly combining 9 features quantifying linear, HOS and nonlinear/complex dynamics (see Table 3), we achieved a balanced accuracy of $79.17 \%$ (with specificity $83.33 \%$ and sensitivity of $75.00 \%$ ) discriminating at a singlesubject level. Of note, the selected feature-set comprised features coming from both standard frequency domain and high order spectra in addition to the fractal dimension and recurrence plot indexes. This result is in agreement with a large amount of studies suggesting that an effective, comprehensive assessment of ANS activity through cardiovascular dynamics should include multivariate, linear and nonlinear measures (see, e.g., reviews $[65,72]$ and references therein). To further confirm this, we showed a significant decrease of the accuracy (i.e., 66.67\%) considering only HRV features quantifying cardiovascular linear dynamics. Concerning the learning algorithm comparisons, we showed that the feature-set, characterized by a combination of linear and nonlinear features, was better classified by a model-free nonlinear method (i.e., k-NN). Particularly, this latter outperforms SVM when the features are selected considering a wrapper approach (i.e., a sequential forward selection) rather than estimating possible dependences within the feature set.

The present study came with limitations. First, due to the higher prevalence of dysphoria in the female population, and to avoid gender-related confounding factors, female volunteers only were enrolled $[53,79]$. In this population, we did not control for the ovaric cycle phase which could bias heartbeat dynamics [80,81]. Moreover, clinically depressed patients and individuals with BDI-II score between 8 and 12 were excluded. This was to ensure separation between groups without ambiguity [23, 24]. Finally, we considered short-term cardiovascular variability, leveraging on standard recordings performed during standard cardiological visits, therefore regardless any, may be important, indices of long-term variability. Another limitation of this paper is related to the missing comparison with cardiovascular data gathered from depressed patients. Although we compared with previous evidences reported in the literature, as we found a distinctive cardiovascular pattern for dysphoric individuals, a direct comparison with depressed patients would have given further insights to our conclusion: dysphoria is a distinct mood disorder and not simply a milder form of depression. Finally, a more general limitation regarding the approach applied to the present paper has to be discussed. Despite the large number of publications in this field, the study of 
psychobiology of mental disorders is limited by the fact that patients are selected using subjective measures exclusively (e.g., through answers to a questionnaire and/or to a clinical interview). This criticism seems even more important when artificial intelligence algorithms are used. In fact, the model is usually optimized to fit the subjective, and thus potentially unreliable, measures. Nevertheless, the use of well validated questionnaires and clinical interviews (e.g., the SCID-I) to evaluate patient's symptoms is, to date, the "gold standard of psychological assessment". Thus, the only way to currently study biological correlates of mental disorders is to consider the patient experience as described by clinical rating scales. Note that rating scales, and in particular BDI-II, have a good reliability and several external and internal validations [30,31]. Our aim was not to implement a model of HRV describing dysphoric patients, but to use learning algorithms to better describe and understand dysphoria. Indeed, through this approach, we were able to uncover novel properties of this pathological condition.

Although with intrinsic limitations, this study provides important insights in the psychophysiology of a mental disorder such as dysphoria. Once the characterization of associated autonomic dysfunctions will be well-defined, future studies may apply unsupervised learning algorithms identify sub-clusters of patients different symptoms or clinical phenotypes [82].

To conclude, to the best of our knowledge this is the first study investigating multi-dimensional feature of ANS dynamics of cardiovascular control in dysphoria with statistical power at the singlesubject level. This multi-feature approach, not only produces a better characterization of the disorder from a physiological point of view, but also highlights novel properties of autonomic activity in dysphoric participants. This latter, in turn, provides further strength to the idea, supported by experimental studies [76, 77], that dysphoria is a defined condition with specific characteristics which are different both from healthy fully euthimic controls and from full-blown major depression. Moreover, ANS multidimensional analysis may result in a more specific clinical tool than the ones relying on a single feature. Given the aspecific dynamics associated with ANS functioning, very similar changes of a single heartbeat feature, in fact, may be observed in pathological conditions, as well as in healthy ones (e.g., during cognitive/physical stress, sleep).

Future endeavours will be directed to increase the number of dysphoric and healthy control subjects enrolled in the study, including male population, as well as the investigation of further nonlinear/complexity measures of heartbeat dynamics to increase the sensitivity and specificity of the proposed assessment.

\subsection{Acknowledgments}

The research leading to these results has received funding from the European Commission Horizon2020 Programme under grant agreement no. 689691 "NEVERMIND". 


\section{REFERENCES}

[1] R. C. Kessler and E. J. Bromet, "The epidemiology of depression across cultures," Annual review of public health, vol. 34, pp. 119-138, 2013.

[2] K. Sunagawa, T. Kawada, T. Nakahara, "Dynamic nonlinear vago-sympathetic interaction in regulating heart rate". Heart and Vessels 13, no. 4, pp. 157?174, 1998.

[3] P. Sobocki, B. Jönsson, J. Angst, and C. Rehnberg, "Cost of depression in europe." The journal of mental health policy and economics, vol. 9, no. 2, pp. 87-98, 2006.

[4] C. J. Murray and A. D. Lopez, "Evidence-based health policy-lessons from the global burden of disease study," Science, vol. 274, no. 5288, p. 740, 1996.

[5] C. J. Murray, A. D. Lopez et al., Global burden of disease. Harvard University Press Boston, 1996, vol. 1.

[6] S. Bădescu, C. Tătaru, L. Kobylinska, E. Georgescu, D. Zahiu, A. Zăgrean, and L. Zăgrean, "The association between diabetes mellitus and depression," Journal of Medicine and Life, vol. 9, no. 2, p. 120, 2016.

[7] S. J. Leistedt, P. Linkowski, J. P. Lanquart, J. E. Mietus, R. B. Davis, A. L. Goldberger, M. D. Costa, "Decreased neuroautonomic complexity in men during an acute major depressive episode: analysis of heart rate dynamics." Translational psychiatry, 1(7), e27, 2011.

[8] J. A. Blumenthal, H. S. Lett, M. A. Babyak, W. White, P. K. Smith, D. B. Mark, R. Jones, J. P. Mathew, M. F. Newman, N. Investigators et al., "Depression as a risk factor for mortality after coronary artery bypass surgery," The Lancet, vol. 362, no. 9384, pp. 604-609, 2003.

[9] M. M. Burg, C. M. Benedetto, and R. Soufer, "Depressive symptoms and mortality two years after coronary artery bypass graft surgery (cabg) in men," Psychosomatic Medicine, vol. 65, no. 4, pp. 508-510, 2003.

[10] R. M. Carney, K. E. Freedland, G. E. Miller, and A. S. Jaffe, "Depression as a risk factor for cardiac mortality and morbidity: a review of potential mechanisms," Journal of psychosomatic research, vol. 53, no. 4, pp. 897-902, 2002.

[11] R. M. Carney, J. A. Blumenthal, P. K. Stein, L. Watkins, D. Catellier, L. F. Berkman, S. M. Czajkowski, C. Oconnor, P. H. Stone, and K. E. Freedland, "Depression, heart rate variability, and acute myocardial infarction," Circulation, vol. 104, no. 17, pp. 2024-2028, 2001.

[12] B. W. Penninx, "Depression and cardiovascular disease: epidemiological evidence on their linking mechanisms," Neuroscience \& Biobehavioral Reviews, 2016.

[13] P. Cuijpers and F. Smit, "Excess mortality in depression: a meta-analysis of community studies," Journal of affective disorders, vol. 72, no. 3, pp. 227-236, 2002.

[14] A. P. Association et al., Diagnostic and statistical manual of mental disorders (DSM-5®). American Psychiatric Pub, 2013.

[15] S.-C. Park, J.-M. Kim, T.-Y. Jun, M.-S. Lee, J.-B. Kim, H.-W. Yim, and Y. C. Park, "How many different symptom combinations fulfil the diagnostic criteria for major depressive disorder? results from the crescend study," Nordic journal of psychiatry, vol. 71, no. 3, pp. 217-222, 2017.

[16] D. A. Regier, W. E. Narrow, D. E. Clarke, H. C. Kraemer, S. J. Kuramoto, E. A. Kuhl, and D. J. Kupfer, "DSM-5 field trials in the united states and canada, part II: test-retest reliability of selected categorical diagnoses," American journal of psychiatry, vol. 170, no. 1, pp. 59-70, 2013.

[17] M. Landén, B. Wennerblom, H. Tygesen, K. Modigh, K. Sörvik, et al., "Heart rate variability in premenstrual dysphoric disorder." Psychoneuroendocrinology, vol. 29, no. 6, pp. 733-740, 2004.

[18] H. B. Mokros, "Communication and psychiatric diagnosis: Tales of depressive moods from two contexts," Health Communication, vol. 5, no. 2, pp. 113-127, 1993.

[19] P. Cuijpers, N. Vogelzangs, J. Twisk, A. Kleiboer, J. Li, and B. W. Penninx, "Differential mortality rates in major and subthreshold depression: meta-analysis of studies that measured both," The British Journal of Psychiatry, vol. 202, no. 1, pp. 22-27, 2013. 
[20] P. Haaf, M. Ritter, L. Grize, M. E. Pfisterer, M. J. Zellweger, B. study group et al., "Quality of life as predictor for the development of cardiac ischemia in high-risk asymptomatic diabetic patients," Journal of Nuclear Cardiology, pp. 1-11, 2017.

[21] O. Mittag, H. Kampling, E. Farin, and P. J. Tully, "Trajectories of depressive symptoms after a major cardiac event," Health psychology open, vol. 3, no. 1, p. 2055102915624873, 2016.

[22] A. J. Carroll, R. Auer, L. A. Colangelo, M. R. Carnethon, D. R. Jacobs Jr, J. C. Stewart, R. Widome, J. J. Carr, K. Liu, and B. Hitsman, "Association of the interaction between smoking and depressive symptom clusters with coronary artery calcification: The cardia study," Journal of Dual Diagnosis, no. just-accepted, pp. 00-00, 2017.

[23] S. Messerotti Benvenuti, G. Buodo, R. Mennella, and D. Palomba, "Somatic, but not cognitiveaffective, symptoms are associated with reduced heart rate variability in individuals with dysphoria," Frontiers in psychology, vol. 6, 2015.

[24] S. Messerotti Benvenuti, R. Mennella, G. Buodo, and D. Palomba, "Dysphoria is associated with reduced cardiac vagal withdrawal during the imagery of pleasant scripts: Evidence for the positive attenuation hypothesis," Biological psychology, vol. 106, pp. 28-38, 2015.

[25] R. Mennella, S. Messerotti Benvenuti, G. Buodo, and D. Palomba, "Emotional modulation of alpha asymmetry in dysphoria: results from an emotional imagery task," International Journal of Psychophysiology, vol. 97, no. 2, pp. 113-119, 2015.

[26] L. L. Judd, M. H. Rapaport, M. P. Paulus, and J. L. Brown, "Subsyndromal symptomatic depression: a new mood disorder?" The Journal of clinical psychiatry, 1994.

[27] S. Messerotti Benvenuti, G. Buodo, and D. Palomba, "Appetitive and aversive motivation in dysphoria: A time-domain and time-frequency study of response inhibition," Biological Psychology, vol. 125, pp. 12-27, 2017.

[28] L. L. Judd, H. S. Akiskal, and M. P. Paulus, "The role and clinical significance of subsyndromal depressive symptoms (ssd) in unipolar major depressive disorder," Journal of affective disorders, vol. 45 , no. 1 , pp. 5-18, 1997.

[29] M. Hamilton, "A rating scale for depression," Journal of Neurology, Neurosurgery \& Psychiatry, vol. 23, no. 1, pp. 56-62, 1960.

[30] A. T. Beck, R. A. Steer, and G. K. Brown, "Beck depression inventory-ii," San Antonio, vol. 78, no. 2, pp. 490-8, 1996.

[31] M. Ghisi, G. Flebus, A. Montano, E. Sanavio, and C. Sica, "Beck depression inventory," Adattamento Italiano: Manuale, Giunti Organizzazioni Speciali, Florence, Italy, 2006.

[32] M. First, R. Spitzer, M. Gibbon, and J. Williams, "Stuructured clinical interview for dsm-iv axis i disorders (scid-i), clinical version. washington dc and london," 1997.

[33] R. Carney, K. Freedland, and R. Veith, "Depression, the autonomic nervous system, and coronary heart disease," Psychosomatic Medicine, vol. 67, no. SUPPL. 1, pp. S29-S33, 2005.

[34] W. Kop, P. Stein, R. Tracy, J. Barzilay, R. Schulz, and J. Gottdiener, "Autonomic nervous system dysfunction and inflammation contribute to the increased cardiovascular mortality risk associated with depression," Psychosomatic Medicine, vol. 72, no. 7, pp. 626-635, 2010.

[35] M. Jarrett, R. Burr, K. Cain, V. Hertig, P. Weisman, and M. Heitkemper, "Anxiety and depression are related to autonomic nervous system function in women with irritable bowel syndrome," Digestive Diseases and Sciences, vol. 48, no. 2, pp. 386-394, 2003.

[36] Z. Bajk, C.-C. Szekeres, K. Kovcs, K. Csap, S. Molnr, P. Soltsz, E. Nyitrai, M. Magyar, L. Olh, D. Bereczki, and L. Csiba, "Anxiety, depression and autonomic nervous system dysfunction in hypertension," Journal of the Neurological Sciences, vol. 317, no. 1-2, pp. 112-116, 2012.

[37] M. Hu, F. Lamers, E. De Geus, and B. Penninx, "Differential autonomic nervous system reactivity in depression and anxiety during stress depending on type of stressor," Psychosomatic Medicine, vol. 78, no. 5, pp. 562-572, 2016.

[38] A. H. Kemp, D. S. Quintana, M. A. Gray, K. L. Felmingham, K. Brown, and J. M. Gatt, "Impact of depression and antidepressant treatment on heart rate variability: a review and meta-analysis," Biological psychiatry, vol. 67, no. 11, pp. 1067-1074, 2010. 
[39] K. Udupa, T. Sathyaprabha, J. Thirthalli, K. Kishore, G. Lavekar, T. Raju, and B. Gangadhar, "Alteration of cardiac autonomic functions in patients with major depression: a study using heart rate variability measures," Journal of affective disorders, vol. 100, no. 1, pp. 137-141, 2007.

[40] K. G. Van der Kooy, H. P. van Hout, H. W. van Marwijk, M. de Haan, C. D. Stehouwer, and A. T. Beekman, "Differences in heart rate variability between depressed and non-depressed elderly," International journal of geriatric psychiatry, vol. 21, no. 2, pp. 147-150, 2006.

[41] S. Boettger, D. Hoyer, K. Falkenhahn, M. Kaatz, V. K. Yeragani, and K.-J. Bär, "Nonlinear broad band dynamics are less complex in major depression," Bipolar disorders, vol. 10, no. 2, pp. 276-284, 2008.

[42] G. Valenza, M. Nardelli, G. Bertschy, A. Lanata, and E. Scilingo, "Mood states modulate complexity in heartbeat dynamics: A multiscale entropy analysis," EPL (Europhysics Letters), vol. 107, no. 1, p. 18003, 2014.

[43] V. K. Yeragani, K. R. K. Rao, M. R. Smitha, R. B. Pohl, R. Balon, and K. Srinivasan, "Diminished chaos of heart rate time series in patients with major depression," Biological psychiatry, vol. 51, no. 9, pp. 733-744, 2002.

[44] E. Nahshoni, D. Aravot, D. Aizenberg, M. Sigler, G. Zalsman, B. Strasberg, S. Imbar, E. Adler, and A. Weizman, "Heart rate variability in patients with major depression," Psychosomatics, vol. 45, no. 2, pp. 129-134, 2004.

[45] G. Valenza, L. Citi, C. Gentili, A. Lanatá, E. Scilingo, and R. Barbieri, "Point-process nonlinear autonomic assessment of depressive states in bipolar patients," Methods of Information in Medicine, vol. 53, no. 4, pp. 296-302, 2014.

[46] K. Chua, V. Chandran, U. Acharya, and C. Lim, "Computer-based analysis of cardiac state using entropies, recurrence plots and poincare geometry," Journal of medical engineering $\mathcal{G}^{3}$ technology, vol. 32, no. 4, pp. 263-272, 2008.

[47] M. Masè, L. Faes, R. Antolini, M. Scaglione, and F. Ravelli, "Quantification of synchronization during atrial fibrillation by shannon entropy: validation in patients and computer model of atrial arrhythmias," Physiological measurement, vol. 26, no. 6, p. 911, 2005.

[48] J. P. Zbilut, N. Thomasson, and C. L. Webber, "Recurrence quantification analysis as a tool for nonlinear exploration of nonstationary cardiac signals," Medical engineering 8 physics, vol. 24, no. 1, pp. 53-60, 2002.

[49] D. Hubert, D. Mestivier, J. Jarnet, M. E. Safar, and N. P. Chau, "Quantification of sympathetic and parasympathetic tones by nonlinear indexes in normotensive rats," American Journal of Physiology-Heart and Circulatory Physiology, vol. 275, no. 4, pp. 1290-1297, 1998.

[50] Jarosław Piskorski and Przemysław Guzik. Filtering poincare plots. Computational methods in science and technology, 11(1):39-48, 2005.

[51] Yoav Benjamini and Yosef Hochberg. Controlling the false discovery rate: a practical and powerful approach to multiple testing. Journal of the royal statistical society. Series B (Methodological), pages 289-300, 1995.

[52] G. Valenza, A. Greco, L. Citi, M. Bianchi, R. Barbieri, and E. Scilingo, "Inhomogeneous pointprocesses to instantaneously assess affective haptic perception through heartbeat dynamics information," Scientific Reports, vol. 6, 2016.

[53] M. M. Weissman and G. L. Klerman, "Sex differences and the epidemiology of depression," Archives of general psychiatry, vol. 34, no. 1, pp. 98-111, 1977.

[54] R. Lynn and T. Martin, "Gender differences in extraversion, neuroticism, and psychoticism in 37 nations," The Journal of social psychology, vol. 137, no. 3, pp. 369-373, 1997.

[55] J. M. Cyranowski, E. Frank, E. Young, and M. K. Shear, "Adolescent onset of the gender difference in lifetime rates of major depression: a theoretical model," Archives of general psychiatry, vol. 57, no. 1, pp. 21-27, 2000.

[56] K. S. Kendler, L. M. Thornton, and C. A. Prescott, "Gender differences in the rates of exposure to stressful life events and sensitivity to their depressogenic effects," American Journal of 
Psychiatry, vol. 158, no. 4, pp. 587-593, 2001.

[57] I. H. Gotlib and C. L. Hammen, Handbook of depression. Guilford Press, 2008.

[58] R. D. Goodwin and I. H. Gotlib, "Gender differences in depression: the role of personality factors," Psychiatry research, vol. 126, no. 2, pp. 135-142, 2004.

[59] L. Lasa, J. Ayuso-Mateos, J. Vazquez-Barquero, F. Diez-Manrique, and C. Dowrick, "The use of the beck depression inventory to screen for depression in the general population: a preliminary analysis," Journal of affective disorders, vol. 57, no. 1, pp. 261-265, 2000.

[60] S. D. Sprinkle, D. Lurie, S. L. Insko, G. Atkinson, G. L. Jones, A. R. Logan, and N. N. Bissada, "Criterion validity, severity cut scores, and test-retest reliability of the beck depression inventory-ii in a university counseling center sample." Journal of counseling psychology, vol. 49, no. 3 , p. $381,2002$.

[61] C. D'Angelo, A. Mirijello, L. Leggio, A. Ferrulli, V. Carotenuto, N. Icolaro, A. Miceli, V. D'Angelo, G. Gasbarrini, and G. Addolorato, "State and trait anxiety and depression in patients with primary brain tumors before and after surgery: 1-year longitudinal study," 2008.

[62] J. J. Gross and O. P. John, "Individual differences in two emotion regulation processes: implications for affect, relationships, and well-being." Journal of personality and social psychology, vol. 85, no. 2, p. 348, 2003.

[63] J. Pan and W. J. Tompkins, "A real-time QRS detection algorithm," IEEE transactions on biomedical engineering, no. 3, pp. 230-236, 1985.

[64] L. Citi, E. N. Brown, and R. Barbieri, "A real-time automated point-process method for the detection and correction of erroneous and ectopic heartbeats," IEEE transactions on biomedical engineering, vol. 59, no. 10, pp. 2828-2837, 2012.

[65] U. R. Acharya et al., "Heart rate variability," in Advances in cardiac signal processing. Springer, 2007, pp. 121-165.

[66] U. R. Acharya, K. P. Joseph, N. Kannathal, C. M. Lim, and J. S. Suri, "Heart rate variability: a review," Medical and biological engineering and computing, vol. 44, no. 12, pp. 1031-1051, 2006.

[67] D. E. Lake, J. S. Richman, M. P. Griffin, and J. R. Moorman, "Sample entropy analysis of neonatal heart rate variability," American Journal of Physiology-Regulatory, Integrative and Comparative Physiology, vol. 283, no. 3, pp. R789-R797, 2002.

[68] D. E. Lake and J. R. Moorman, "Accurate estimation of entropy in very short physiological time series: the problem of atrial fibrillation detection in implanted ventricular devices," American Journal of Physiology-Heart and Circulatory Physiology, vol. 300, no. 1, pp. H319-H325, 2011.

[69] K. Ansari-Asl, G. Chanel, and T. Pun, "A channel selection method for eeg classification in emotion assessment based on synchronization likelihood," in Signal Processing Conference, 2007 15th European. IEEE, 2007, pp. 1241-1245.

[70] J. M. Mendel, "Tutorial on higher-order statistics (spectra) in signal processing and system theory: theoretical results and some applications," Proceedings of the IEEE, vol. 79, no. 3, pp. 278-305, 1991.

[71] I. Guyon, J. Weston, S. Barnhill, and V. Vapnik, "Gene selection for cancer classification using support vector machines," Machine learning, vol. 46, no. 1, pp. 389-422, 2002.

[72] R. Sassi et al., "Advances in heart rate variability signal analysis: joint position statement by the e-cardiology esc working group and the european heart rhythm association co-endorsed by the asia pacific heart rhythm society," Europace, p. euv015, 2015.

[73] C. Gentili, S. Messerotti Benvenuti, D. Palomba, A. Greco, E. P. Scilingo, and G. Valenza, "Assessing mood symptoms through heartbeat dynamics: An HRV study on cardiosurgical patients", Journal of Psychiatric Research, vol. 95, pp. 179-188, 2017.

[74] A. Porta et al., "Short-term complexity indexes of heart period and systolic arterial pressure variabilities provide complementary information," Journal of Applied Physiology, vol. 113, no. 12 , pp. $1810-1820,2012$.

[75] T. Makikallio et al., "Abnormalities in beat to beat complexity of heart rate dynamics in patients 
with a previous myocardial infarction," Journal of the American College of Cardiology, vol. 28, no. 4, pp. 1005-1011, 1996.

[76] V. Starcevic, D. Berle, K. Viswasam, A. Hannan, D. Milicevic, V. Brakoulias, and E. Dale, "Specificity of the relationships between dysphoria and related constructs in an outpatient sample," Psychiatric Quarterly, vol. 86, no. 4, pp. 459-469, 2015.

[77] A. Dayer, J.-M. Aubry, L. Roth, S. Ducrey, and G. Bertschy, "A theoretical reappraisal of mixed states: dysphoria as a third dimension," Bipolar Disorders, vol. 2, no. 4, pp. 316-324, 2000.

[78] I. H Gotlib and J. Joormann, "Cognition and depression: current status and future directions". Annual review of clinical psychology, vol 6, pp. 285-312, 2010.

[79] R. C. Kessler, "Epidemiology of women and depression," Journal of affective disorders, vol. 74, no. 1, pp. 5-13, 2003.

[80] X. Bai, J. Li, L. Zhou, and X. Li, "Influence of the menstrual cycle on nonlinear properties of heart rate variability in young women," American Journal of Physiology-Heart and Circulatory Physiology, vol. 297, no. 2, pp. H765-H774, 2009.

[81] A. S. Leicht, D. A. Hirning, and G. D. Allen, "Heart rate variability and endogenous sex hormones during the menstrual cycle in young women," Experimental physiology, vol. 88, no. 3, pp. 441446, 2003.

[82] C. Gentili, "Why do we keep failing in identifying reliable biological markers in depression?" Journal of Evidence-Based Psychotherapies, vol. 17, no. 2, pp. 69-84, 2017. 